\title{
A HaemSTAR is born; a trainee-led, UK-wide research network in haematology
}

There is an associated link between clinical research engagement and improved patient outcomes. ${ }^{1}$ Not only does this apply to the subjects of research but also to those patients who are not active participants but are treated at sites where research is conducted. ${ }^{2}$ Unfortunately, clinical research activity is not uniformly spread across the country. It is centred around tertiary referral centres and medical schools. ${ }^{3}$ Thus, patients treated outside these larger hospitals are not receiving the associated benefits of clinical research. Not only is health research not fairly distributed geographically but activity also differs significantly by specialty. Even within specialties, like haematology, where there is a plethora of clinical research, there is under-representation of non-malignant conditions when compared to trial activity in haematological malignancies. ${ }^{4}$

An additional problem is the bureaucracy involved in clinical research. ${ }^{5}$ This reinforces the geographical imbalance because the lack of research infrastructure in smaller hospitals makes it difficult to set up studies.

To address these issues in the long term, it is essential to encourage ubiquitous clinician engagement in research. Therefore, research skills must be embedded into medical training and the bureaucratic process of running clinical research studies needs to be made less burdensome. To achieve this, in 2017 the National Institute for Health Research (NIHR) National Specialty Group in Haematology set up a trainee-led research network called HaemSTAR (Haematology Specialty Trainee Audit and Research; Fig 1).

HaemSTAR has a central committee who prioritise studies to promote. There is a lead trainee and associated consultant mentor in each region. Thus, the regional leads have sources of both national and local support and also have efficient lines of communication with all other trainees in that region. Their role is to rapidly disseminate information and coordinate regional activity and local trainee involvement as is required. In addition, they continually add to a list of frequently encountered issues for each study that is published on the HaemSTAR website (https://HaemSTAR.org). Thus, the whole group can learn from individual experiences and make subsequent study activity at new sites more efficient.

Examples of completed collaborative projects are shown in Table 1. They include mass participation and huge recruitment in our national 'flash-mob' audit and rapid opening and completion of phase III and IV studies in the little-investigated disease area of immune thrombocytopenia. All projects were conducted across teaching and district general hospitals. This has led to national awards for group members and interest from disease specific study groups and the pharmaceutical industry who want to engage in future collaboration. It must be recognised that this model cannot work without incentives, thus HaemSTAR's policy is that people who commit time and effort to projects are given citable

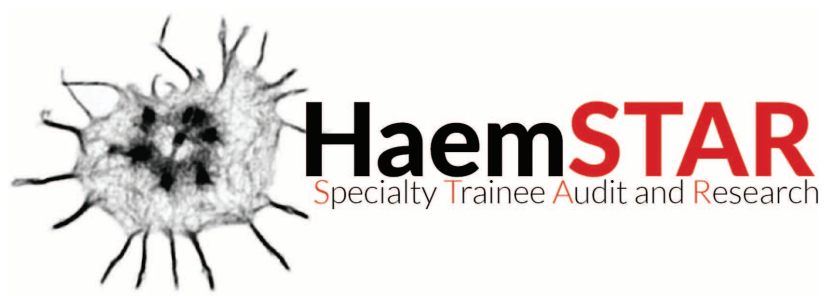

Fig 1. HaemSTAR logo.

collaborator status on any publications that come out of the research.

The aim in writing this article is to make this novel and successful trainee-led model more widely known such that other specialties can copy it for the benefit of patients in all specialties across the UK.

\section{Additional information}

For further information on HaemSTAR please visit https:// HaemSTAR.org or follow @HaemSTAR UK on Twitter.

\section{Acknowledgements}

We wish to thank the NIHR Haematology NSG who have provided logistical and financial support in addition to Rosaria Alexandre and Charlie Clegg from Brandcast Media for their expertise in the digital promotion of HaemSTAR. We are also grateful to the chief investigators of TRAIT, FLIGHT and the ITP Registry studies (Dr Nichola Cooper, Dr Charlotte Bradbury and Dr Vickie McDonald, respectively). We would finally like to thank Natalie Poulter from the University of Birmingham for providing the microscopy image of actin in a spreading platelet for use in our logo.

Phillip LR Nicolson

Clinical lecturer in haematology, HaemSTAR, NIHR Haematology Clinical Research Network, University of Birmingham, Birmingham,

UK and University Hospitals Birmingham NHS Foundation Trust,

Birmingham, UK

Michael JR Desborough Consultant haematologist, HaemSTAR, NIHR Haematology Clinical Research Network and NIHR Oxford Bioresource, Oxford, UK

Daniel Hart

Consultant haematologist, NIHR Haematology Clinical Research Network and Barts and the London School of Medicine and Dentistry, Queen Mary University of London, London, UK 


\begin{tabular}{|c|c|c|c|c|c|}
\hline Study & Type of study & $\begin{array}{l}\text { Total number } \\
\text { of sites }\end{array}$ & $\begin{array}{l}\text { HaemSTAR } \\
\text { supported sites (\%) }\end{array}$ & $\begin{array}{l}\text { Total number } \\
\text { of patients }\end{array}$ & $\begin{array}{l}\text { HaemSTAR supported } \\
\text { patient recruitment (\%) }\end{array}$ \\
\hline MASCOT (pilot) & Retrospective & 6 & $6(100 \%)$ & 31 & $31(100 \%)$ \\
\hline FLIGHT & $\begin{array}{l}\text { Phase III prospective } \\
\text { randomised controlled trial }\end{array}$ & 39 & $17(44 \%)$ & 124 & $54(44 \%)$ \\
\hline TRAIT & Phase IV retrospective & 15 & $8(53 \%)$ & 268 & $104(39 \%)$ \\
\hline Flash-mob audit & Retrospective national audit & 39 & $39(100 \%)$ & 978 & $978(100 \%)$ \\
\hline
\end{tabular}

Tina T Biss

Consultant haematologist, NIHR Haematology Clinical Research Network and Newcastle upon Tyne Hospitals NHS Foundation Trust, Newcastle upon Tyne, UK

Gillian C Lowe

Consultant haematologist, NIHR Haematology Clinical Research Network and University Hospitals Birmingham NHS Foundation Trust, Birmingham, UK

Cheng-Hock Toh

Consultant haematologist, NIHR Haematology Clinical Research Network and Royal Liverpool University Hospital, Liverpool, UK

\section{References}

1 Jonker L, Fisher SJ. The correlation between National Health Service trusts' clinical trial activity and both mortality rates and care quality commission ratings: a retrospective cross-sectional study. Public Health 2018;157:1-6.
2 Downing A, Morris E], Corrigan N et al. High hospital research participation and improved colorectal cancer survival outcomes: a population-based study. Gut 2017;66:89-96.

3 Toh $\mathrm{CH}$. The current geographical spread of research is failing patients. BMJ 2019.

4 https://clinicaltrials.gov. Search term: 'haematology'. Filtered for trials that were open and actively recruiting. [Accessed 12 April 2018].

5 Rule S, LeGouill S. Bureaucracy is strangling clinical research. BM] 2019;364:I1097.

Address for correspondence: Dr Phillip Nicolson, Institute of Cardiovascular Sciences, University of Birmingham, Vincent Drive Edgbaston, Birmingham B15 2TT, UK. Email: p.nicolson@bham.ac.uk 